Doi: $10.32481 /$ djph.2021.12.007

\title{
The Genetic Testing Stewardship Program:
}

\section{A Bridge to Precision Diagnostics for the Non-genetics Medical Provider}

Morgan Thomas, MGC, CGC; Louise Amlie-Wolf, MS, CGC; Laura Baker, MGC, CGC; Karen W. Gripp, MD, FACMG

Nemours Children's Hospital

\begin{abstract}
Genetic/genomic testing can reveal important diagnostic information, provided the appropriate test is chosen and the results are interpreted accurately. Choosing an informative, cost-effective genetic testing strategy is a complex process. Nemours' Genetic Testing Stewardship Program (GTSP) is a genetic counselor-staffed consultative service serving the Nemours Children's Health $(\mathrm{NCH})$ system. This program assists non-genetics providers and their patients access genetic testing for improved patient care and reduced costs. GTSP genetic counselors provide pre- and post-test genetic counseling, obtain informed consent, ensure complete documentation, and provide results interpretation/disclosure support for ordering providers. Implementation of this program began with genetic testing needs assessments and presentations about this novel service at subspecialty division meetings. GTSP expanded at NCH Delaware and NCH Orlando to include review of inpatient genetic testing for medical necessity. GTSP has experienced success from provider satisfaction and cost savings perspectives. GTSP has produced cost savings of $>\$ 400,000$, supporting financial sustainability of the program. Additionally, genetic counselors bill for visits, creating the foundation for a future revenue stream which is likely to increase as reimbursement for genetic counseling services improves. GTSP has become integral in the $\mathrm{NCH}$ system by improving patient access to genetic testing in a safe, efficient, effective manner.
\end{abstract}

\section{Introduction}

Nemours Children's Health created the Genetic Testing Stewardship Program (GTSP) in July 2018 as part of the Precision Medicine initiative. The objectives of GTSP include providing genetic counseling support to non-genetics providers while reducing inappropriate genetic test orders and ensuring the medical utility of ordered genetic testing. GTSP genetic counselors offer pre- and post-test genetic counseling, obtain informed consent, and interpret/disclose test results.

The GTSP is a consultative genetic counseling service with utilization management components. An on-call genetic counselor reviews genetic test requests received during business hours and assesses the medical necessity of the requested test. The genetic counselor then reviews relevant patient history and discusses a recommended genetic testing strategy with the ordering provider to reach a mutually agreed upon plan. When a testing strategy is selected, the genetic counselor provides pre-test genetic counseling, if requested, and obtains informed consent (which is required by Delaware State law) before coordinating the sample collection. The genetic counselor will also ensure proper documentation of counseling, informed consent, and results in the electronic medical record, and will provide direct patient care for results disclosure when needed. Additionally, GTSP reviews all orders for inpatient genetic testing and provides 
intervention, in addition to the above-described services, when testing is deemed not medically necessary for the admission.

\section{Methods}

\section{Place and Time}

GTSP was initiated at Nemours Children's Hospital Delaware in Wilmington, Delaware in 2018 (previously known as Nemours/Alfred I. duPont Hospital for Children) and currently operates at multiple sites including Nemours Children's Hospital, Delaware, Delaware Valley satellite clinics, and Nemours Children's Hospital, Florida.

\section{Population}

This intervention was targeted towards non-genetics clinicians ordering genetic testing at Nemours Children's Hospital Delaware and aims to increase diagnostic yield and appropriate test utilization for the pediatric population.

\section{Purpose}

Non-genetics providers often order genetic testing independent of a trained genetics healthcare provider. However, literature suggests an increased error rate in genetic testing ordered without genetics support. ${ }^{1}$ The Medical Genetics workforce nationally is not sufficient to provide genetic services for all individuals requiring genetic testing. ${ }^{2}$ The risks associated with erroneous test ordering are numerous, and include reduced clinical relevance, missed diagnoses, incorrect interpretation and increased harm to patients due to improper patient care. ${ }^{1}$ Risks can be mitigated by including genetic counselors in genetic testing utilization management, which can significantly reduce costs to a healthcare system ${ }^{1}$ when inappropriate or incorrect orders are modified or cancelled.

Genetic counselors are healthcare providers who provide education about genetic conditions and genetic testing to patients. ${ }^{3}$ This information is provided to help patients and their families understand the risks, benefits and limitations of genetic testing in order to make an informed decision about proceeding with a specific genetic test. GTSP genetic counselors partner with non-genetics physicians, advanced practice nurses, and physician assistants to determine if genetic testing is necessary for a patient, and if so, what genetic testing approach is most appropriate and cost effective. Providers often know which patients would benefit from genetic testing, but are limited by their understanding of evolving testing technologies, access to and experience with laboratories offering testing, and understanding of legal requirements regarding informed and documented consent for genetic testing. Furthermore, providers often lack the administrative support to coordinate ordering, insurance coverage, and billing, as well as to the follow up on results.

Genetic counselors, along with a genetic counseling assistant, can support each of these steps. This allows patients to receive medically indicated genetic testing and genetic counseling ordered by non-genetics providers without a referral to the Medical Genetics Department, which often has a long wait list and may not be needed, depending on the indication for testing.

Lastly, GTSP genetic counselors offer pre- and post-test genetic counseling support to families undergoing genetic testing. The support and information that genetic counselors provide is 
integral in helping patients through the decision-making process for genetic testing, as well as during and after results disclosure. Involving genetic counselors in informed consent discussions leads to increased patient knowledge about the testing they receive. ${ }^{3}$ Additionally, genetic counseling performed by a genetic counselor allows providers more time to focus on a patient's medical subspecialty care without the additional burden of discussing genetic testing details during their appointment. Genetic counselors are uniquely suited to improve patient care in pediatric healthcare.

\section{Implementation}

The precision medicine initiative at Nemours aims to tailor healthcare based on an individuals' genetic and health factors. Staff from medical genetics and genetic counseling recognized the potential value of a genetic testing stewardship program and worked with Nemours leadership at the medical and executive levels to secure funding through the Precision Medicine Initiative. This program design was based loosely on the very successful antibiotic stewardship program. Subsequently, a needs assessment was completed with hospital subspecialty divisions to ensure that the program met the varying needs of providers in different specialties, and to build relationships with the various stakeholders.

Original staffing for the program included a part time $(0.5$ FTE) genetic counselor manager and a full-time genetic counselor. Oversight for the program is provided by an American College of Medical Genetics board-certified medical geneticist and pediatrician. During the implementation phase, GTSP staff offered educational presentations to hospital divisions to promote awareness of this new service. The program was well received, and quickly expanded to include a genetic counseling assistant, who is integral in managing the clerical workload associated with genetic testing, scheduling, and patient communication tasks. Additional genetic counseling staff was hired at the equivalent of one additional full time genetic counselor and an interdepartmental partnership was formed with a part-time $(0.5 \mathrm{FTE})$ genetic counselor dedicated to and funded by pediatric neurology. As of 2021, the GTSP employs the equivalent of 2.0 full time genetic counselors, 0.5 FTEs of genetic counseling manager, one fulltime genetic counseling assistant and 0.5 FTEs of neurology-funded specialty genetic counselor.

\section{Results}

During the first year of the program, electronic health record (EHR) tools were built to increase provider ease of access to the program and to allow for genetic test order review by the GTSP staff. When the program was initiated, providers could request program assistance in inpatient and outpatient settings through EHR order, email, phone or staff message. In the following year, GTSP added the automatic review of all inpatient genetic test orders for medical appropriateness and cost effectiveness. A 3-tier system to filter these orders was developed in partnership with the Epic EHR development team. Tier 1 tests are molecular tests that may fall outside the GTSP staff expertise, such as HLA typing or pharmacogenomic testing. Tests categorized as tier 2 are automatically routed to the GTSP inbox for review but do not require approval for ordering, such as Factor V Leiden testing and chromosome microarray. Tier 3 classification was reserved for high-cost tests with a high risk for result misinterpretation and high complexity consent process. These tests require approval by a GTSP counselor before ordering and trigger a corresponding EHR alert. This automated review process allowed for identification of high-risk cases and 
optimization of cost savings opportunities. Attention to relationship building and clinical partnership has led to an overwhelmingly positive reception from providers.

Nemours is a multistate healthcare system, and as staffing for GTSP has increased, the program has extended telehealth services to satellite clinics in PA, NJ, and DE. The program continues to grow and support cross campus collaboration with Nemours Children's Hospital, Florida.

\section{Evaluation}

Since its inception in the last quarter of 2018, GTSP genetic counselors assisted with and reviewed testing for $\sim 1900$ cases. About $25 \%$ were completed in the inpatient setting and $75 \%$ in the outpatient setting. When genetic testing was ordered, the genetic counselor provided pre- and post-test counseling in $79 \%$ of cases. This indicates a major shift in the burden of this work from the ordering provider to the genetic counseling team. The introduction of this service has been met with universal enthusiastic support by Nemours providers. A provider satisfaction survey distributed to Nemours providers utilizing the GTSP service from 2018 to 2020 revealed that providers were significantly more $(\mathrm{p}<0.0001$; $\mathrm{OR}=86.1)$ comfortable with all aspects of the genetic testing and counseling process when in partnership with counselors from GTSP, and that almost all $(n=41 / 45)$ were satisfied or very satisfied with the GTSP service. Forty-two of the 45 providers indicated that the program had a positive impact on their practice. No providers indicated a negative impact on their practice. When asked to describe their experience with the service in their own words, providers indicated that they were more willing to consider genetic testing for their patients with help from GTSP, that the program made genetic testing more accessible, and that the program allows for valuable genetic counseling services. One provider went so far as to call the program a "game changer for me in my practice." It is clear that from the perspective of Nemours healthcare providers, the implementation of GTSP has been an unambiguous success.

In addition to these qualitative provider facing improvements, GTSP aids in utilization management efforts by evaluating cases for cost effectiveness and necessity. Modifications to requested testing strategies were recommended in approximately $15 \%$ and cancellations of testing occurred approximately $25 \%$. The most common reasons for modification of testing strategy were identification of a more cost-effective test or an improvement to the requested testing strategy based on the patient's clinical presentation. Approximately $10 \%$ of cases were cancelled due to errors, lack of medical necessity, or requests for duplicated testing. Other reasons for cancellation included deferral of genetic testing for further evaluation (medical genetics or other diagnostic studies necessary to determine proper testing strategy), patient lost to follow up, and families declining testing after proper counseling. In total, alterations to testing (including modifications and cancellations) led to an estimated $\$ 380,000$ in savings in 2019. This excludes testing not completed due to lack of family follow-up. An additional $\$ 87,000$ of potential revenue captured was through insurance prior authorizations facilitated by GTSP.

Longitudinal analysis of GTSP case data has shown that the program has been successful in increasing access and proper utilization of genetic testing, allowing for proper consenting and counseling of patients, and reducing potential errors and unnecessary healthcare spending to a significant degree. 
Doi: $10.32481 /$ djph.2021.12.007

\section{Adverse Effects}

Implementation of the GTSP at Nemours highlighted inequities in access to genetics services and provided an opportunity to increase equity in access. Genetics services are typically more readily available at major academic medical centers, which may be prohibitive for individuals living in remote or rural communities. As Nemours is the only children's hospital in the State of Delaware, this information is particularly applicable to Nemours. To combat this inequity, the GTSP has expanded their telehealth program to assist providers seeing patients in satellite clinics throughout the Delaware valley, and the service area of the program now extends to include Pennsylvania, New Jersey, Maryland, and in the future, Florida.

Though there have been few patient facing adverse effects relating to the implementation of the genetic testing stewardship program at Nemours, opportunities for provider education, improvements to the electronic medical record, and areas for increased compliance with state consent laws have been identified and are an active area of focus for the program.

\section{Sustainability}

The GTSP is largely self-sustaining, as the program led to an estimated $\$ 400,000+$ in cost savings to the Nemours system annually. In addition to cost savings, genetic counseling is a billable service under its own CPT code. Because genetic counselors are not currently recognized by the Centers for Medicare and Medicaid as reimbursable providers, reimbursement for this code varies widely. Impending changes to federal legislation may make billable genetic counseling encounters a more reliable source of revenue. It is a future goal of the program to evaluate revenue generation in addition to cost savings produced by the program.

\section{Public Health Significance}

In 2015, the United States Precision Medicine Initiative was announced with the long-term goal of expanding precision medicine to all areas of healthcare. In alignment with this goal, Nemours Children's Health System prioritized the establishment of the Genetic Testing Stewardship Program. Access to genetic testing is an increasingly important component of precision medicine with implications for the diagnosis and treatment of rare and common disease. However, in $2015,30 \%$ of genetics practices reported a wait time of over three months for a new patient visit; a steep increase from $10 \%$ of clinics reporting a three month wait time in $2005 .^{2}$ This concerning trend highlights a severe access deficit for genetics services. The GTSP and non-genetic subspecialist partnership allows for expedient identification and testing of individuals with high suspicion for genetic disease, as well as appropriate triage to a medical geneticist as necessary. On average, patients referred to GTSP are seen within 20 days of referral, and same day or next day services are available for time-sensitive indications. Programs such as the GTSP could significantly increase access to necessary diagnostic testing and genetic counseling services throughout the healthcare system.

In addition to the access benefits that the GTSP has shown, genetic counselor involvement in genetic test utilization contributes to significant healthcare savings, increased quality of patient care and reduction of diagnostic errors. Currently, over 75,000 genetic testing products and an estimated 10,000 unique forms of testing are currently available to the medical community on a clinical basis. ${ }^{4}$ With this oversaturation of testing choices, providers are often overwhelmed when determining an appropriate genetic test for their patients. Previous studies have shown that 
providers without specialty training in genetics often make errors when ordering genetic testing and often are underequipped to provide accurate and comprehensive interpretation of results. ${ }^{5}$ This can lead to healthcare waste, and, most importantly, missed or misinterpreted diagnoses. Genetic counselors are uniquely trained to navigate the complex landscape of genetic testing and assist non-genetics providers in making the most effective diagnostic decisions for their patients.

\section{Conclusion}

The GTSP model demonstrates a highly effective and sustainable framework for integrating next generation genetic and genomic diagnostics into pediatric specialty care. This may translate into other areas of healthcare and should be considered by institutions intending to prioritize genetic and genomic diagnostics in primary and specialty care. This approach utilizes the combined expertise of pediatric subspecialists and genetic counselors to increase access to genetics services, improve quality of patient care, and reduce unnecessary healthcare spending.

These authors can be contacted at genetic.testing@nemours.org.

\section{References}

1. Mathias, P. C., Conta, J. H., Konnick, E. Q., Sternen, D. L., Stasi, S. M., Cole, B. L., .. . Dickerson, J. A. (2016, August). Preventing genetic testing order errors with a laboratory utilization management program. American Journal of Clinical Pathology, 146(2), 221-226. PubMed https://doi.org/10.1093/ajcp/aqw105

2. Maiese, D. R., Keehn, A., Lyon, M., Flannery, D., \& Watson, M., \& the Working Groups of the National Coordinating Center for Seven Regional Genetics Service Collaboratives. (2019, August). Current conditions in medical genetics practice. Genet Med, 21(8), 1874 1877. PubMed https://doi.org/10.1038/s41436-018-0417-6

3. Madlensky, L., Trepanier, A. M., Cragun, D., Lerner, B., Shannon, K. M., \& Zierhut, H. (2017, June). A rapid systematic review of outcome studies in genetic counseling. Journal of Genetic Counseling, 26(3), 361-378. PubMed https://doi.org/10.1007/s10897-017-0067-X

4. Conway, M. E., Kalejta, C. D., Sternen, D. L., \& Singh, I. R. (2020, March 9). The importance of genetics experts in optimizing genetic test orders through prospective and retrospective reviews. American Journal of Clinical Pathology, 153(4), 537-547. PubMed https://doi.org/10.1093/ajcp/aqz188

5. Haga, S. B., Kim, E., Myers, R. A., \& Ginsburg, G. S. (2019, May 24). Primary care physicians' knowledge, attitudes, and experience with personal genetic testing. Journal of Personalized Medicine, 9(2), 29. PubMed https://doi.org/10.3390/jpm9020029

Copyright (c) 2021 Delaware Academy of Medicine / Delaware Public Health Association.

This is an Open Access article distributed under the terms of the Creative Commons Attribution Non-Commercial License (https://creativecommons.org/licenses/by-nc-nd/4.0/) which permits unrestricted non-commercial use, distribution, and reproduction in any medium, provided the original work is properly cited. 